\title{
MACROFAUNA DA LITEIRA EM SISTEMAS AGROFLORESTAIS SOBRE PASTAGENS ABANDONADAS NAAMAZÔNIA CENTRAL
}

\author{
Sandra C. TAPIA-CORAL ${ }^{1}$, Flávio J. LUIZÃO', Elisa V. WANDELLI ${ }^{2}$
}

RESUMO - As densidades (ind $\left./ \mathrm{m}^{2}\right)$ e biomassas $\left(\mathrm{mg} / \mathrm{m}^{2}\right)$ de grupos selecionados da macrofauna da liteira (isópodos, diplópodos e cupins) foram estudadas sob diferentes sistemas agroflorestais (SAFs) implantados em áreas de pastageńs degradadas e abandonadas na Amazônia central. O estudo foi feito na Estação de Pesquisa Agroflorestal da EMBRAPA/CPAA, $70 \mathrm{~km}$ ao norte de Manaus, usando-se três blocos ao acaso, com cinco tratamentos, em quinze parcelas de $3000 \mathrm{~m}^{2}$; cada bloco tinha quatro diferentes tipos de SAFs e uma capoeira (CAP), usada como controle, onde se manteve a regeneração natural existente. OS SAFs eram dois sistemas agrossilviculturais, um com base em palmeiras e outro mais diversificado, denominado multiestrato, e dois sistemas agrossilvipastoris, de altos e baixos insumos. Em cada parcela, foram coletadas 50 amostras da macrofauna da liteira, usando-se um quadro de madeira de 50 x $50 \mathrm{~cm}$, nas estaçōes chuvosa e seca. A densidade e biomassa da macrofauna foram maiores no SAF mais diversificado (multiestrato) e geralmente maiores sob liteiras de cupuaçu e palmeiras, destacando-se o grupo dos isópodos com as maiores densidades e biomassas, seguido pelos diplópodos, nos SAFs, e pelos cupins, na capoeira. Todos os SAFs apresentaram maiores densidades e biomassas da macrofauna do que a capoeira, indicando a formação de micro-sítios favoráveis à sua ação, devido à melhor qualidade da liteira produzida e/ou da abundância localizada de liteiras de menor qualidade produzida pelas diversas espécies plantadas. Os resultados sugerem a melhoria do funcionamento dos processos de reciclagem no solo e, assim, das perspectivas de sustentabilidade da produção.

Palavras chaves: macrofauna da liteira, biomassa de invertebrados, sistemas agroflorestais

Litter-Layer Macrofauna in Agroforestry Systems on Abandoned Pastures in Central Amazonia ABSTRACT - The densities (ind $\left./ \mathrm{m}^{2}\right)$ and biomasses $\left(\mathrm{mg} / \mathrm{m}^{2}\right)$ of selected groups of the macrofauna in the litter layer (Isopoda, Diplopoda and Isoptera) under different agroforestry systems (SAFs) planted in areas of degraded and abandoned pastures in central Amazonia, were investigated. The study was carried out at the Agroforestry Research Station of EMBRAPA/ CPAA, located $70 \mathrm{~km}$ north of Manaus, using three blocks with five treatments at random in fifteen plots of $3000 \mathrm{~m}^{2}$; each block had four different types of SAFs and a second-growth area (CAP), used as control, where existing natural regeneration was allowed to grow. Of the two agrosilvicultural systems, one was based on palm trees and the other more diversified, socalled multistrata; and of the two agrosilvipastoril systems, one had high and the other low inputs of fertilizer. In each plot, 50 samples of litter macrofauna were collected, using a $50 \mathrm{x}$ $50 \mathrm{~cm}$ wooden frame, in the rainy and dry seasons. The density and biomass of the litter macrofauna were higher in the most diversified SAF (multistrata), and generally higher under "cupuaçu" and palm tree litters. Isopoda had the highest densities and biomasses, followed by Diplopoda in the SAFs and by termites in the second growth. All SAFs presented macrofauna densities and biomasses higher than the second growth, indicating the creation of favorable habitats, due to either a higher quality of the litter produced or a higher abundance of localized litter of lower quality. The results suggest an improved functioning of the nutrient recycling processes in the soil, and sustainable productivity prospects of the agroforestry systems, especially the most diversified ones.

Key-words: litter macrofauna, invertebrate biomass, agroforestry systems.

1 Coordenação de Pesquisas em Ecologia, Instituto Nacional de Pesquisas da Amazônia. Caixa Postal 478, 69011-970 Manaus, AM. E-mail: fluizao@inpa.gov.br ${ }^{2}$ CPAA/EMBRAPA, Manaus, AM. 


\section{INTRODUÇÃO}

Nos sistemas florestais nativos, a superfície do solo está geralmente coberta por detritos orgânicos, principalmente de origem vegetal, coletivamente chamados de liteira (Marino et al., 1980). Esta representa uma fonte de energia e de nutrientes para toda uma comunidade de organismos que realiza sua decomposição, liberando os nutrientes minerais e transformando em húmus as substâncias orgânicas mais refratárias à decomposição (Anderson \& Swift, 1983). A diversidade e abundância das comunidades de macroinvertebrados do solo e da liteira e a relativa importância de grupos principais desta fauna podem ser usadas como indicadoras da qualidade do solo (Stork \& Eggleton, 1992). Entre os fatores biológicos que controlam e diferenciam sazonalmente a decomposição e/ou remoção da liteira sobre o solo, destacam-se a penetração das raizes finas superficiais no material em decomposição e a atividade de remoção dos macro-artrópodos do solo e da liteira, especialmente dos cupins ou térmitas (Luizão \& Schubart, 1987; Luizão, 1995), os quais têm sido considerados, junto com as formigas, como os grupos de maior biomassa da fauna do solo na Amazônia central (Fittkau \& Klinge 1973; Bandeira \& Harada, 1991, 1998). No entanto, outros grupos de macroinvertebrados que habitam a liteira e a camada superficial do solo podem ter uma importância e biomassa superiores às que lhes têm sido atribuidas; este parece ser o caso dos diplópodos e dos isópodos na
Amazônia central (Harada \& Bandeira, 1994; Ribeiro, 1994; Luizão, 1995). Os diplópodos, conhecidos como "milipedes" ou "embuás", são detritivoros terrestres e eventualmente decompositores de folhas de liteira (Hopkin \& Read, 1992) e de outros materiais, incluindo as fezes de mamíferos herbívoros (Dindal, 1990). Os isópodos, conhecidos como "piolhos de madeira" ou "tatuzinhos", são crustáceos terrestres e estão também entre os principais decompositores da matéria orgânica, sendo conhecidos como cavadores de solos geralmente encontrados em florestas primárias (Muchmore, 1990).

Além do metabolismo químico, os organismos têm um papel mecânico no ciclo de nutrientes: perfuram o solo, madeiras vivas e mortas, cortam e raspam folhas e, assim, multiplicam as superficies para $o$ ataque dos microrganismos decompositores e patogênicos sobre a matéria orgânica (Walker \& Franken, 1983).

A população dos artrópodos do solo da floresta é perturbada pela derrubada da floresta seguida pela queima da vegetação, que é uma forma comum de preparo da área, tanto para agricultura como para pecuária, na Amazônia brasileira. Isto provoca a morte de grande quantidade de animais do solo, alterando deste modo a diversidade faunística da área (Oliveira \& Franklin, 1993; Lavelle et al., 1995); no entanto, a biomassa vegetal que não foi queimada, principalmente os grandes troncos, mantém áreas com menor perturbação que funcionam como refúgios temporários para a fauna do solo (Teixeira \& Schubart, 1988; Oliveira \& Franklin, 1993). 
Comparações entre a floresta natural e áreas de pastagens mostraram que a crescente compactação do solo, causada pelo pastoreio, pode afetar a intensidade e a qualidade das atividades da microflora e microfauna, devido à diminuição da aeração e oxigenação do solo (Luizão et al., 1992). Esta pode ser uma das causas da falta de sustentabilidade das pastagens na Amazônia brasileira, levando ao seu subseqüente abandono.

Para ser sustentável, a substituição da floresta primária deve ser executada de modo a criar um sistema de cultivo com características o mais próximas possiveis das do ecossistema original (Magalhães, 1979). Por outro lado, nas áreas já abandonadas, as capoeiras manejadas e as plantações de árvores e arbustos podem contribuir significativamente para a recuperação da produtividade e da biodiversidade do solo de áreas desmatadas e/ou degradadas e, assim, o uso de sistemas agroflorestais poderia contribuir para este objetivo (Fernandes et al., 1994a; b).

O objetivo do presente estudo foi o de estimar as densidades (ind $/ \mathrm{m}^{2}$ ) e biomassas $\left(\mathrm{mg} / \mathrm{m}^{2}\right)$ de grupos selecionados da macrofauna da liteira (especialmente isópodos, diplópodos e cupins) sob diferentes sistemas agroflorestais implantados em áreas de pastagens degradadas e abandonadas.

\section{MATERIAIS E MÉTODOS}

\section{Localização e caracterização das áreas de estudo}

$\mathrm{O}$ estudo foi desenvolvido na Estação Experimental do CPAA/ EMBRAPA-Manaus (Centro de Pesquisa Agroflorestal da Amazônia
Ocidental/Empresa Brasileira de Pesquisa Agropecuária). Esta situa-se no Distrito Agropecuário da SUFRAMA, ao norte de Manaus, no km 54 da BR-174 (Manaus-Boa Vista), área compreendida entre as coordenadas geográficas $2^{\circ} 31^{\prime}$ a $2^{\circ} 32^{\prime}$ $\mathrm{S}$ e $60^{\circ} 01^{\prime}$ a $60^{\circ} 02^{\prime} \mathrm{W}$. O clima da região é caraterizado como do tipo Ami pela classificação de Köppen (Ribeiro \& Adis, 1984), com uma média anual de pluviosidade entre 1500 e $2500 \mathrm{~mm}$. Em 1997, ano do presente estudo, os dados de clima e temperatura foram registrados na Estação Experimental da CEPLAC (Comissão Executiva do Plano da Lavoura Cacaueira, situada $5 \mathrm{~km}$ a sudoeste da área de estudo), verificandose uma precipitação anual de $2167,5 \mathrm{~mm}$, variando de 20,5 a $407 \mathrm{~mm}$ por mês. Houve um periodo de chuvas fortes e frequentes antes da primeira coleta (durante a estação chuvosa) e um período bem seco antecedendo a segunda coleta (estação seca) (Fig. 1). A temperatura média foi de $26,2^{\circ} \mathrm{C}$, com média das mínimas de 22,4 ${ }^{\circ} \mathrm{C}$ e das máximas de $32,8^{\circ} \mathrm{C}$. A média mensal da umidade relativa do ar variou de 81 a $90 \%$, com uma média anual de $83,9 \%$, caracterizando a micro-região como 'tropical úmida bastante chuvosa'.

A área de estudo está situada sobre um extenso platô de Latossolo Amarelo distrófico (Sanchez, 1976; Teixeira \& Bastos, 1989), textura muito argilosa, onde são encontradas limitações de fertilidade, acidez elevada, baixa capacidade de troca de cátions, deficiências de fósforo, nitrogênio, cálcio e magnésio (Teixeira \& Bastos, 1989; Mackerrow, 1992; 


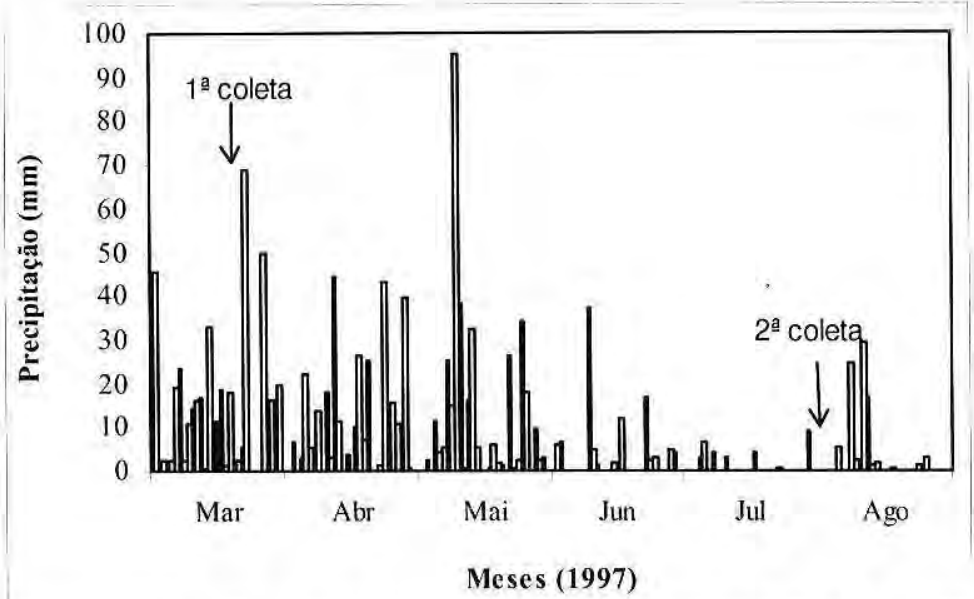

Figura 1. Precipitação pluviométrica diária durante o periodo de estudo. As setas indicam as datas das coletas da macrofauna (março e agosto de 1997).

Fernandes \& Matos, 1995), e alta saturação de alumínio (Tab, 1).

\section{Delineamento Experimental}

O delineamento estabelecido pelo CPAA/EMBRAPA consiste em três blocos ao acaso, com cinco tratamentos, em parcelas de $3000 \mathrm{~m}^{2}$ $(60 \mathrm{~m} \times 50 \mathrm{~m})$. Em cada bloco, foram usados quatro tipos diferentes de sistemas agroflorestais (SAFs) e uma parcela controle, onde se manteve a regeneração natural (capoeira). Os tratamentos agroflorestais, com espécies variadas (Apêndice 1), consistem em: Sistema Agrossilvicultural 1 (AS1): tem por base palmeiras e fruteiras perenes; Sistema Agrossilvicultural 2 (AS2): denominado "multiestrato", com aproveitamento dos recursos de produção em diferentes etapas durante o desenvolvimento das espécies florestais e várias fruteiras perenes; Sistema Agrossilvipastoril 1 (ASP1) e Sistema Agrossilvipastoril 2 (ASP2), têm composição e arranjo

Tabela 1. $\mathrm{pH}$, concentrações de $\mathrm{P}$ assimilável, $\mathrm{K}, \mathrm{Ca}$ e $\mathrm{Mg}$ disponíveis, e saturação de $\mathrm{Al}$ do solo nos sistemas agroflorestais e no controle, nos anos de 1991 e 1996. Os tratamentos, na primeira coluna, correspondem a: AS1 e AS2 = sistemas agrossilviculturais 1 (baseado em palmeiras arbóreas) e 2 (multiestrato); ASP1 e ASP2 = sistemas agrossilvipastoris 1 (com altos insumos) e 2 (baixos insumos); $\mathrm{CAP}=$ capoeira $($ controle $)$.

\begin{tabular}{|c|c|c|c|c|c|c|c|c|c|c|c|c|}
\hline \multirow{3}{*}{$\begin{array}{l}\text { Tratam. } \\
\text { (anos) }\end{array}$} & \multirow[b]{3}{*}{1991} & \multicolumn{2}{|l|}{$\mathrm{pH}$} & & \multicolumn{2}{|l|}{ K } & \multicolumn{2}{|l|}{$\mathrm{Ca}$} & \multicolumn{2}{|l|}{$\mathrm{Mg}$} & \multirow{3}{*}{$\begin{array}{c}\text { Sat. Al } \\
\% \\
1996\end{array}$} \\
\hline & & & & $\mathrm{mg} / \mathrm{kg}$ & & & …........ &.. $\mathrm{cmol}_{\circ}$ & $\mathrm{kg} . . . . .$. & $\ldots \ldots \ldots$. & & \\
\hline & & 1996 & 1991 & 1996 & 1991 & 1996 & 1991 & 1996 & 1991 & 1996 & 1991 & \\
\hline AS 1 & 4,4 & 4,5 & 2,2 & 3,0 & 0,14 & 0,08 & 1,27 & 0,54 & 0,47 & 0,24 & 34,6 & 54,9 \\
\hline AS 2 & 4,3 & 4,4 & 1,7 & 2,8 & 0,13 & 0,09 & 0,86 & 0,36 & 0,31 & 0,17 & 49,7 & 65,2 \\
\hline ASP 1 & 4,3 & 4,3 & 1,9 & 1,6 & 0,12 & 0,05 & 0,87 & 0,50 & 0,33 & 0,12 & 48,5 & 63,5 \\
\hline ASP 2 & 4,3 & 4,3 & 1,6 & 1,6 & 0,10 & 0,05 & 0,75 & 0,24 & 0,33 & 0,15 & 53,1 & 74,2 \\
\hline CAP & 4,5 & 4,3 & 2,6 & 1,2 & 0,20 & 0,07 & 1,27 & 0,42 & 0,52 & 0,31 & 34,6 & 56,1 \\
\hline
\end{tabular}

Fonte: EMBRAPA/CPAA, 1997. Relatório anual. 
semelhantes entre si, diferenciando-se quanto ao manejo e preparo do solo: o ASP1 é baseado no conceito de altos insumos ("high input"), enquanto que o ASP2 é baseado no conceito de baixos insumos ("low input"). As espécies de árvores usadas em ambos os sistemas foram ingá, mogno e paricá; nas parcelas do ASP1 foi estabelecida a leguminosa forrageira Desmodium ovalifolium Wall. consorciada com Brachiaria brizantha (Höchst) Stapf), enquanto que no ASP2 foi estabelecida a leguminosa $D$. ovalifolium consorciada com Brachiaria humidicola (Rendle) Schweinckt) (Wandelli et al., 1997). Nestes tratamentos, em janeiro e março de 1997 (estação chuvosa), houve o ingresso do gado por um período de sete dias cada vez, sendo colocadas cinco cabeças de gado no ASP1 e três cabeças no ASP2 (Wandelli et al., 1997). Todos os SAFs têm cerca viva de Gliricidia sepium (Jacq.) Walp (Leguminosae), que é podada duas vezes por ano e espalhada como adubo verde pelas parcelas dos SAFs; além disso, a biomassa das capinas das plantas invasoras é também espalhada nas entre-linhas dos SAFs. A regeneração natural ou capoeira (CAP) foi usada como controle, sendo pouco diversificada e formada principalmente por espécies de plantas pioneiras como Vismia sp. (Matos et al., 1996; Wandelli et al., 1997).

\section{Metodologia das coletas no campo e identificação da macrofauna}

Em cada uma das quinze parcelas (cinco tratamentos em três blocos) foram coletadas ao acaso 50 amostras da macrofauna da liteira, usando-se um quadro de madeira de $50 \times 50 \mathrm{~cm}$ e uma faca. Cada amostra era depositada em uma grande bandeja plástica. Para retirar a macrofauna da liteira (somente os animais > $2 \mathrm{~mm}$ ) (Lavelle, 1988; Lavelle et al., 1995) foi feita uma catação manual no próprio local da coleta, com ajuda de uma pinça (principalmente para os predadores). Os animais coletados foram conservados em frascos plásticos com álcool a 70\% e levados ao laboratório para posterior identificação.

A macrofauna da liteira foi separada e identificada com ajuda de uma lupa, pinças e com o auxílio de chaves e catálogos publicados (Coinneau, 1974; Muchmore, 1990; Nutting, 1990; Hoffman et al., 1996), a nivel de ordem e, quando possível, familia. Depois de identificada, foi ordenada em grupos principais, como Isopoda, Diplopoda, Isoptera e "outros artrópodos". Estes foram então pesados em balança analítica (após enxugados em papel absorvente), para se determinar sua biomassa úmida em álcool (Dindal, 1990).

Os dados obtidos foram transformados com o propósito de homogeneizar a variância (Zar, 1974), usandose a raiz quadrada como fator de transformação para a densidade e biomassa da macrofauna. Foram feitas análises de variância, seguidas de testes de Tukey para avaliar as diferenças entre os tratamentos e entre as vegetações predominantes dentro dos sistemas agroflorestais (palmeiras, cupuaçu e entre-linhas (espaço entre as fileiras de arvores plantadas)) no AS1; árvores, cupuaçu e entre-linhas no AS2; árvores, Desmodium e Brachiaria no ASP1 e 2; e capoeira no tratamento controle (CAP). 


\section{RESULTADOS}

\section{Macrofauna da liteira nos sistemas agroflorestais e vegetações predominantes}

Na estação chuvosa, um total de quinze grupos taxonômicos de macroinvertebrados foi encontrado nos SAFs e no controle, sendo o grupo numericamente predominante o dos isópodos. Sua densidade $\left(85,9 \mathrm{ind} / \mathrm{m}^{2}\right)$ no AS2 foi maior do que nos demais tratamentos (Tab. 2 e Apêndice 2). O total de indivíduos da macrofauna foi significativamente maior nos sistemas agrossilviculturais AS1 (fruteiras perenes) e AS2 (multiestrato) do que no ASP1 (agrossilvipastoril - altos insumos), ASP2 (agrossilvipastoril - baixos insumos) e controle (CAP) (ANOVA; $\mathrm{F}=18,1$; $\mathrm{P}<0,001)$. As densidades de animaais encontrados no chão dominado por liteira de cupuaçu, palmeiras e das entre-linhas foram maiores do que sob a liteira das demais espécies ( $\mathrm{F}=16,5 ; \mathrm{P}<0,001)$ (Fig. 2). $\mathrm{Na}$ estação seca, um total de treze grupos taxonômicos foi encontrado nos SAFs, sendo novamente os isópodos os invertebrados mais abundantes, com uma densidade maior no AS2 $\left(50,5 \mathrm{ind} / \mathrm{m}^{2}\right)$ do que nos demais tratamentos (Tab. 2 e Apêndice 3); as maiores densidades da macrofauna total foram outra vez encontradas no AS1 e AS2 ( $F=14,5$; $\mathrm{P}<0,001)$. Em relação à cobertura do solo, as densidades encontradas nos locais em que o chão era dominado por liteira das palmeiras e de cupuaçu foram maiores do que sob a liteira das demais espécies vegetais $(\mathrm{F}=11,5 ; \mathrm{P}<0,001)$ (Fig. 2), O total geral da macrofauna da liteira foi significativamente maior na estação chuvosa do que na seca $(F=80,6$; $\mathrm{P}<0,001$ ), principalmente no tratamento AS2 (multiestrato), composto por uma maior diversidade de espécies plantadas.

A biomassa úmida em álcool da macrofauna, na estação chuvosa, foi significativamente maior no AS1 e AS2 do que nos demais tratamentos (ANOVA; $\mathrm{F}=12,4 ; \mathrm{P}<0,001$ )(Tab. 3). As biomassas encontradas nas entre-

Tabela 2. Densidade da macrofauna da liteira (ind $/ \mathrm{m}^{2}$ ), nas estações chuvosa e seca, nos sistemas agroflorestais e no controle. Os valores representam a média de três blocos $(n=3)$, e os erros padrões das médias estão representados entre parênteses, Valores seguidos por letras diferentes nas colunas indicam diferenças significativas ao nivel de $0,1 \%(P<0,001)$ entre os tratamentos. Os símbolos dos tratamentos são os mesmos explicados na Tabela 1.

\begin{tabular}{|c|c|c|c|c|c|c|}
\hline \multicolumn{7}{|c|}{ Densidade da macrofauna (ind $/ \mathrm{m}^{2}$ ) } \\
\hline Tratamentos & Diplopoda & Isopoda & $\begin{array}{c}\text { Estação chuvosa } \\
\text { Isoptera }\end{array}$ & Outros & $\mathrm{N}^{\circ}$ Grupos & Total \\
\hline AS1 & $1,15( \pm 1,09)$ a & $51,1( \pm 40,2) a$ & $0,85( \pm 0,0) a$ & $4,01( \pm 1,89)$ a & 14 & $57,1 \mathrm{a}$ \\
\hline AS2 & $4,22( \pm 1,03) b$ & $85,9( \pm 13,3) a$ & $0,85( \pm 0,0) a$ & $4,43( \pm 1,02)$ a & 11 & $95,4 \mathrm{a}$ \\
\hline ASP1 & $1,39( \pm 1,32) \mathrm{a}$ & $21,2( \pm 20,2) b$ & $0( \pm 0,0) \mathrm{a}$ & $1,41( \pm 0,10) b$ & 13 & $24,0 \mathrm{~b}$ \\
\hline ASP2 & $1,57( \pm 0,59)$ a & $29,2( \pm 20,1) b$ & $0( \pm 0,0)$ a & $3,13( \pm 0,83)$ a & 12 & $33,9 b$ \\
\hline CAP & $0,67( \pm 0,31) \mathrm{a}$ & $13,9( \pm 7,17) \mathrm{b}$ & $4,96( \pm 0,07) \mathrm{b}$ & $0,27( \pm 0,03) b$ & 9 & $19,8 \mathrm{~b}$ \\
\hline Tratamentos & Diplopoda & Isopoda & $\begin{array}{c}\text { Estaçăo seca } \\
\text { Isoptera }\end{array}$ & Outros & $\mathrm{N}^{\circ}$ Grupos & Total \\
\hline AS1 & $0,22( \pm 1,1) \mathrm{a}$ & $40,0( \pm 21,1) a$ & $3,07( \pm 1,1)$ a & $1,71( \pm 1,1)$ a & 9 & $45,0 \mathrm{a}$ \\
\hline AS2 & $0,24( \pm 0,02) \mathrm{a}$ & $50,5( \pm 25,3) a$ & $4,21( \pm 2,1)$ a & $1,95( \pm 1,2) \mathrm{a}$ & 10 & 56,9 a \\
\hline ASP1 & $0,03( \pm 0,0)$ a & $9,9( \pm 3,3) b$ & $0( \pm 0,0) b$ & $0,67( \pm 0,32) b$ & 8 & $10,6 \mathrm{~b}$ \\
\hline ASP2 & $0( \pm 0,0) \mathrm{a}$ & $11,2( \pm 9,2) b$ & $0,13( \pm 0,1) b$ & $0,77( \pm 0,31) b$ & 6 & $12,1 \mathrm{~b}$ \\
\hline CAP & $0,06( \pm 0,0)$ a & $16,0( \pm 12,1) b$ & $0,61( \pm 0,3) b$ & $0,03( \pm 0,0) b$ & 5 & $16,7 b$ \\
\hline
\end{tabular}



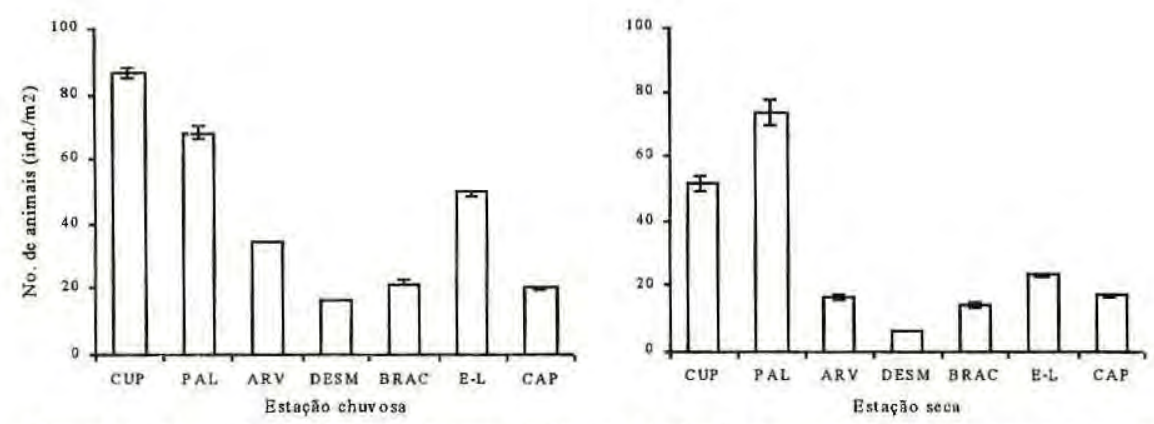

Figura 2. Densidade da macrofauna da liteira (ind $/ \mathrm{m}^{2}$ ) nas estações chuvosa e seca, sob as vegetações predominantes de Cupuaçu (CUP), Palmeiras (PAL), Árvores plantadas (ARV), Desmodium (DESM), Brachiaria (BRAC), Entre-Linhas (E-L) e Capoeira (CAP) dentro dos sistemas agroflorestais e no controle. Os valores são médias de três blocos $(n=3)$ e as barras finas verticais representam o erro padrão da média.

linhas e nos locais onde o chão era dominado por liteira de cupuaçu e das palmeiras foram maiores do que sob as demais espécies de liteira $(\mathrm{F}=4,0$; $\mathrm{P}<0,05)$ (Fig. 3). Na estação seca, não houve diferenças significativas entre os tratamentos, assim como entre as vegetações predominantes, embora tenha sido observada uma biomassa visivelmente mais alta sob a liteira das palmeiras. A biomassa total da macrofauna foi significativamente maior na estação chuvosa do que na seca $(\mathrm{F}=6,84 ; \mathrm{P}<0,05)$ (Tab. 3).
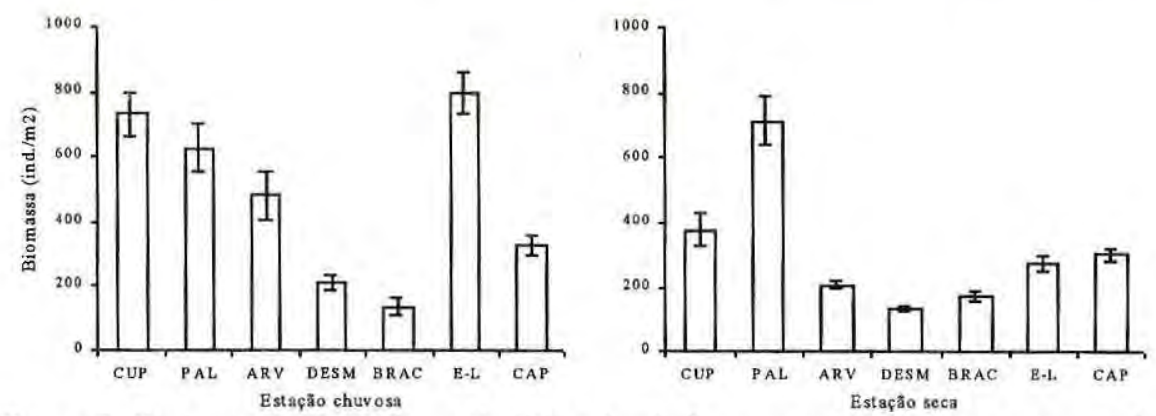

Figura 3. Biomassa da macrofauna da liteira $\left(\mathrm{mg} / \mathrm{m}^{2}\right)$ nas estações chuvosa e seca, sob as vegetações predominantes de Cupuaçu (CUP), Palmeiras (PAL), Árvores (ARV), Desmodium (DESM), Brachiaria (BRAC), Entre-Linhas (E-L) e Capoeira (CAP) nos sistemas agroflorestais e no controle. Os valores são médias de três blocos $(n=3)$ e as barras finas verticais representam o erro padrão da média. 
Tabela 3. Biomassa úmida em álcool da macrofauna da liteira $\left(\mathrm{mg} / \mathrm{m}^{2}\right)$, nas estações chuvosa e seca, nos sistemas agroflorestais e no controle. Os valores representam a média de três blocos $(n=3)$, e os erros padrões das médias estão representados entre parênteses. Valores seguidos por letras diferentes nas colunas indicam diferenças significativas ao nivel de $0,1 \%(P<0,001)$ entre os tratamentos. Os símbolos dos tratamentos são os mesmos explicados na Tabela 1.

\begin{tabular}{|c|c|c|c|c|c|}
\hline \multicolumn{6}{|c|}{ Biomassa da macrofauna (mg/m²) } \\
\hline \multicolumn{6}{|c|}{ Estação chuvosa } \\
\hline Tratamentos & Diplopoda & Isopoda & Isoptera & Outros & Total \\
\hline AS1 & $17,0( \pm 16,9)$ a & $593( \pm 251) a$ & $1,16( \pm 3,47) a$ & $67,5( \pm 66,9)$ a & $679( \pm 318) \mathrm{a}$ \\
\hline AS2 & $103( \pm 86,7) b$ & $785( \pm 556)$ a & $0( \pm 0,0) a$ & $23,6( \pm 13,1) b$ & $912( \pm 604)$ a \\
\hline ASP1 & $18,5( \pm 18,4) \mathrm{a}$ & $169( \pm 144) b$ & $0( \pm 0,0) a$ & $11,6( \pm 20,8) b$ & $199( \pm 165) b$ \\
\hline ASP2 & $18,3( \pm 22,3) a$ & $119( \pm 61,2) b$ & $0( \pm 0,0)$ a & $7,2( \pm 3,9) b$ & $145( \pm 73,7) b$ \\
\hline CAP & $22,3( \pm 18,2)$ a & $288( \pm 185) b$ & $9,07( \pm 22,2) \mathrm{a}$ & $6,5( \pm 9,3) b$ & $326( \pm 190) b$ \\
\hline \multicolumn{6}{|c|}{ Estação seca } \\
\hline Tratamentos & Diplopoda & Isopoda & Isoptera & Outros & Total \\
\hline AS1 & $18,9( \pm 16,0)$ a & $434( \pm 393)$ a & $4,80( \pm 7,17) \mathrm{a}$ & $29,3( \pm 39,5)$ a & $487( \pm 433)$ a \\
\hline AS2 & $10,9( \pm 30,2) \mathrm{a}$ & $277( \pm 255)$ a & $3,29( \pm 9,87)$ a & $11,9( \pm 13,8)$ a & $304( \pm 283) \mathrm{a}$ \\
\hline ASP1 & $0( \pm 0,0) b$ & $108( \pm 102) b$ & $0( \pm 0,0) a$ & $42,8( \pm 20,7) b$ & $151( \pm 105) b$ \\
\hline ASP2 & $0( \pm 0,0) b$ & $78,1( \pm 94,7) b$ & $0( \pm 0,0) \mathrm{a}$ & $61,1( \pm 50,8) b$ & $139( \pm 113) b$ \\
\hline CAP & $1,47( \pm 3,59) b$ & $294( \pm 381) \mathrm{a}$ & $0,27( \pm 0,65)$ a & $8,27( \pm 12,9) \mathrm{a}$ & $304( \pm 394) \mathrm{a}$ \\
\hline
\end{tabular}

ram diretamente proporcionais à massa da camada de liteira sobre o solo, exceto no tratamento AS2, que tinha a maior massa de liteira sobre o solo $\left(4,12 \mathrm{t} \mathrm{ha}^{-1}\right)$ e apresentou também as maiores densidade e biomassa de macrofauna nas duas estações (chuvosa e seca). O tratamento AS1, com a menor quantidade de liteira sobre o solo entre todos os sistemas estudados $\left(3,01 \mathrm{t} \mathrm{ha}^{-1}\right)$, apresentou o segundo maior valor para densidade e biomassa da macrofauna. No entanto, nestes dois sistemas, a umidade do solo e da liteira foi visivelmente maior e houve provavelmente uma maior disponibilidade de alimentos (devido à diversidade e qualidade do material vegetal plantado nesses sistemas); juntas, estas duas características devem ter favorecido a presença da fauna. Os tratamentos agrossilvipastoris (ASP1, altos insumos, e ASP2, de baixos insumos), supostamente deveriam ter uma maior densidade de macrofauna, devido às suas coberturas de Desmodium ovalifolium, uma leguminosa herbácea fixadora de nitrogênio. No entanto, apresentaram densidades da macrofauna muito baixas, em ambas as estações do ano. Em parte, isto poderia ser atribuido ao alto conteúdo de polifenóis do Desmodium (Palm \& Sanchez, 1991; Cantarutti et al., 1996) e à alta relação C/ $\mathrm{N}$ do capim Brachiaria misturado ao Desmodium; ambos, capim e Desmodium, podem também ter baixo conteúdo de nitrogênio (Cantarutti et al., 1996). Mais importante, porém, deve ter sido o uso prévio dos ASPs, por sete dias cada um, em janeiro e março/97 (neste último período, até cinco dias antes da coleta), para pastejo de cinco (ASP1) e três (ASP2) cabeças de gado. Isto pode ter afetado seriamente a colonização da macrofauna nestes sistemas agroflorestais (SAFs), tanto em conseqüência do pisoteio direto como da remoção da massa verde de Desmodium e Brachiaria, o que 
diminuiu sobremaneira a produção de liteira nos meses seguintes. Isto significaria uma influência maior e negativa na macrofauna da estação seguinte (seca), o que de fato parece ter ocorrido.

No presente estudo, as densidades da macrofauna foram relativamente baixas, se comparadas com as de outros trabalhos feitos na região (E. Barros et al., não-publicado). A mais provável razão para isso é que o presente estudo, restringindo-se aos animais da camada de liteira sobre o solo, foi feito em 50 pontos ao acaso dentro de cada parcela de SAFs ainda jovens, que apresentam muitos micro-sítios com pouca ou nenhuma cobertura morta. Esta influência pode ser verificada numa amostragem anterior nos mesmos SAFs do presente estudo, em 1995, usando-se o método TSBF (Anderson \& Ingram, 1993), que inclui camadas de liteira e de solo até $30 \mathrm{~cm}$ de profundidade. Foram encontradas maiores densidades e biomassas da macrofauna nos tratamentos agrossilvipastoris (ASP1 e ASP2); praticamente não se encontrou macrofauna na liteira nos tratamentos agrossilviculturais (AS1 e AS2) (E. Barros et al., dados não-publicados). A mais provável explicação é que, naquela época, os sistemas AS1 e AS2 praticamente ainda não produziam liteira e, conseqüentemente, a camada orgânica sobre o solo era virtualmente inexistente. Por outro lado, nos ASPs 1 e 2, a cobertura verde de Desmodium ovalifolium já produzia bastante liteira, proporcionando melhores condições de umidade do solo e fornecimento de matéria orgânica e nutrientes ao solo (E. Barros, comunicação pessoal). No presente estudo, os sistemas agrossilviculturais, com seis anos de idade e uma considerável produção de liteira, tcriam favorecido um maior número de grupos da macrofauna.

Diplopoda e Isopoda são geralmente considerados como tendo uma baixa biomassa em florestas tropicais (Petersen \& Luxton, 1982); no entanto, Lawrence (1953) considerou os diplópodos provavelmente tão importantes como as minhocas para a decomposição, em algumas florestas da África. Os diplópodos têm uma importante função na regulação dos processos de decomposição e subseqüente ciclagem de nutrientes (Lavelle, 1988), particularmente através de suas interações com os microorganismos do solo (Anderson et al., 1985; Anderson, 1988). Em algumas regiões tropicais, esta importância tem sido verificada, através de sua abundância e biomassa, tanto em ambientes naturais como em alterados.

Numa floresta tropical madura em Seram, os diplópodos contribuíram com $82 \%$ do número total de invertebrados e foram também os animais mais importantes em termos de biomassa (Stork \& Brindell, 1993). Na Amazônia central, Luizão (1995) encontrou freqüências e densidades relativamente altas de diplópodos e isópodos em campinarana e em floresta densa, com maiores valores na campinarana, que tinha um alto conteúdo de húmus na camada superior do solo, o que mantém condições mais apropriadas para este grupo (Raw, 1967). Em outros tipos de vegetação, tais como as florestas inundadas por água mista (Lago Janauari, aproximadamente a $10 \mathrm{~km}$ de Manaus), encontraram-se três famílias de 
diplópodos, sendo a mais abundante a família Polyxenidae (Morais, 1995); em florestas inundadas por água preta (igapós), as familias mais importantes nos troncos foram Siphonophoridae e Polyxenidae e em florestas inundadas por água branca (várzeas) foi a família Polyxenidae (Adis et al., 1996). Nestas florestas periodicamente alagáveis, os diplópodos que sobrevivem à inundação desenvolvem estratégias de sobrevivência em conseqüência de mudanças dos fatores abióticos (Adis, 1997).

Em habitats manejados, Lavelle \& Pashanasi (1989) verificaram uma redução na abundância de diplópodos, o que parece ser um fato comum em certos ambientes tropicais, onde eles são muito afetados pelas mudanças climáticas; a macrofauna tem-se mostrado mais sensível a condições ambientais não favoráveis do que a meso e a microfauna (Lavelle et al., 1995). No entanto, em uma pequena capoeira de 13 anos, crescendo sobre área de floresta derrubada, queimada e logo abandonada, ao norte de Manaus, Ribeiro (1994) registrou os diplópodos entre os seis grupos com maior biomassa, na camada de $0-3,5 \mathrm{~cm}$ do solo. Ainda ao norte de Manaus, Harada \& Bandeira (1994) encontraram uma maior abundância de diplópodos e isópodos em plantios de essências madeireiras do que na floresta nativa. Isto pode indicar que estes dois grupos de invertebrados aumentam de importância em ambientes com plantios arbóreos que mantenham boas condições microclimáticas. Este parece ser o caso no presente estudo, onde os diplópodos e isópodos foram mais abundantes nos sistemas agroflorestais com maior cobertura arbórea. Em sistemas de policultivo e de Bactris gasipaes (pupunha) com cobertura de Pueraria, $30 \mathrm{~km}$ a nordeste de Manaus, Vohland \& Schroth (1998) registraram os diplópodos como o segundo grupo mais importante da fauna de invertebrados com dez famílias, sendo $21 \%$ dos indivíduos da família Pyrgodesmidae; no presente estudo, foram encontradas só cinco familias, sendo a mais abundante a familia Spirostreptidae, especialmente na estação chuvosa. Em uma capoeira antiga, $20 \mathrm{~km}$ ao norte de Manaus, Rodrigues (1986) registrou maior abundância de diplópodos na estação seca do ano, na camada de $0-3,5 \mathrm{~cm}$ do solo. Este resultado difere do encontrado no presente trabalho que, no entanto, se restringiu ao estudo dos invertebrados presentes na camada de liteira sobre o solo, no período diurno. A biomassa de diplópodos na estação seca não diminuiu na liteira das palmeiras, como ocorreu nos locais cobertos por outros tipos de liteira; muito pelo contrário, ela aumentou consideravelmente, uma vez que os diplópodos encontravam-se refugiados nos troncos das palmeiras em decomposição sobre o solo, encontrando-se ali um indivíduo da família Chelodesmidae de grande tamanho e peso, que teve forte influência na biomassa total.

Os isópodos estão entre os principais decompositores da matéria orgânica (Dindal, 1980) e são bons indicadores de microclima e umidade do solo (Edwards, 1974; Decaens et al., 1994), sendo encontrados principalmente em florestas primárias. Nos sistemas agroflorestais do 
presente estudo, foram os invertebrados dominantes em termos de densidade e biomassa, e isto podia ser facilmente visto durante as coletas; no campo, verificavase uma clara preferência deles pelas folhas e cascas de cupuaçu dos sistemas agrossilviculturais, assim como pelos troncos cortados das palmeiras (aparentemente, de rápida decomposição - K. Uguen, comunicação pessoal), que estavam jogados no chão como cobertura morta no sistema AS1.

Em todos os tratamentos $\mathrm{e}$ estações do ano, sempre estiveram presentes "outros invertebrados": Gastropoda, Oligochaeta (minhocas), Homoptera, Hemiptera, Opiliones, Formicidae, Chilopoda, Diptera (larvas), Coleoptera, Thysanoptera e Lepidoptera (larvas). No geral, estes foram mais abundantes na estação chuvosa do que na seca, nos tratamentos AS2 e AS1 (Tab. 2 e Apêndices 2 e 3 ), apresentando maiores densidades na liteira das palmeiras; isto pode indicar a formação de micro-sítios favoráveis para os grupos de invertebrados incluídos nesta classificação, nos SAFs com palmeiras. A densidade relativamente baixa de formigas, que é geralmente alta na liteira e no solo em outros estudos, tanto em florestas nativas como em áreas manejadas (Rodrigues, 1986; Harada \& Bandeira, 1994; Ribeiro, 1994; Morais, 1995; Bandeira \& Harada, 1998), provavelmente deveu-se à metodologia de coleta adotada no presente estudo. A triagem, feita no campo em grandes bandejas plásticas, visivelmente permitia a fuga de muitas formigas, diminuindo consideravelmente o número de animais coletados.
O aumento considerável da biomassa dos "outros artrópodos", na estação seca, nos tratamentos agrossilvipastoris, sob as coberturas de Desmodium (leguminosa forrageira) e Braquiaria (gramínea), deveu-se principalmente à presença de alguns coleópteros (besouros) grandes, os quais são dotados de grande mobilidade e não tão dependentes da umidade, como é o caso dos diplópodos e isópodos, que necessitam de umidade alta para sobreviver (Correia \& Faria, 1997). Em plantios de leguminosas arbóreas no Rio de Janeiro, verificou-se que Coleoptera e Formicidae foram os primeiros colonizadores da liteira nestes sistemas (Correia \& Faria, 1997). Vohland \& Schroth (1998) registraram os coleópteros como o grupo numericamente mais importante entre os invertebrados do solo, com oito famílias presentes nos sistemas de policultivo e de Bactris gasipaes com cobertura de Pueraria na região de Manaus. No presente estudo, outro fator deve ter influenciado a maior densidade de coleópteros durante a estação seca, nos sistemas agrossilvipastoris: as fezes do gado (introduzido por duas semanas em janeiro e março/97) teriam servido, mais tarde, como refúgio e substrato preferencial para este grupo de invertebrados (Decaens et al., 1994).

A diversidade das espécies de liteira depositadas sobre o solo pode ter contribuido para as maiores densidades da macrofauna nos sistemas AS1 e AS2. O tratamento AS2 (multiestrato), com uma maior diversidade de plantas, apresentou uma abundância maior de macrofauna na 
liteira do que os demais tratamentos, nas duas estações, e manteve quase a mesma diversidade (número de grupos) da macrofauna nas duas estações do ano, indicando que, num prazo maior, pode vir a ter uma colonização ainda mais diversificada, com novos grupos da macrofauna. Concordemente, num estudo paralelo, neste tratamento (AS2) foi também encontrada a maior biomassa microbiana (Bio-C) do solo superficial entre os SAFs, sendo esta mais alta sob a liteira de cupuaçu (TapiaCoral et al., 1997). Neste caso, a maior diversidade de espécies plantadas no sistema implicaria em diferentes sistemas radiculares das plantas e seus exsudatos, contribuindo para uma melhor estruturação e aeração do solo, características que favorecem o desenvolvimento microbiano (Luizão \& Luizão, 1991) e, subseqüentemente, da fauna do solo. Um estudo feito em Yurimaguas, Perú (ICRAF, 1996), em sistemas multiestratos e de Bactris gasipaes (pupunha) com cobertura de Pueraria com dez anos de idade, indicou que ambos os sistemas conservaram espécies da macrofauna do solo do sistema original (floresta) e, juntos, forneceram nichos ecológicos para muitos colonizadores exóticos. A maior atividade da macrofauna do solo no sistema multiestrato em Yurimaguas foi atribuída à adição de grandes quantidades de liteira de alta qualidade: uma média anual de 11,5 t/ha foi adicionada, formando uma estrutura heterogênea no sistema multiestrato, que teria contribuído para o estabelecimento e crescimento de certos grupos da fauna (ICRAF, 1996). Embora o presente estudo não tenha investigado também a macrofauna do solo, somente a da liteira, supõe-se que esta reflita em grande parte a macrofauna presente e atuante, durante o período diurno, dentro do solo nas condições de solo úmido (coleta da estação chuvosa) e seco (coleta da estação seca, após vários dias sem chuva).

No presente estudo, os resultados mostrando maiores densidades de indivíduos da macrofauna, nas duas estações, e uma alta biomassa na estação chuvosa nas liteiras de cupuaçu e de palmeiras, não concordaram com os resultados de Vohland \& Schroth (1998) que, amostrando policultivos de quatro anos, próximos a Manaus, registraram uma baixa diversidade de grupos da macrofauna na liteira de cupuaçu. No entanto, isto pode ser devido ao fato de que o sistema estudado por eles encontrava-se ainda muito aberto, com pouca liteira depositada sobre o solo, o que difere do SAF do presente estudo, com considerável cobertura do solo pela liteira de cupuaçu. A biomassa da macrofauna encontrada na liteira de palmeiras foi relativamente alta, praticamente não mudando de uma estação para outra. Este resultado pode indicar que os invertebrados procuram refúgio nos troncos e folhas das palmeiras, espalhados dentro dos sistemas. A segunda coleta da macrofauna foi feita logo após a poda das palmeiras para coleta de frutos, com deposição de folhas e troncos sobre o solo. Talvez essa tenha sido a razão principal da abundância dos invertebrados também na estação seca nos locais com predominância de palmeiras. Dois anos antes, nos mesmos SAFs, 
E. Barros et al. (dados não-publicados) também encontraram uma maior densidade e biomassa da macrofauna no solo embaixo das palmeiras do sistema AS1, na estação seca. Assim, verificou-se que a produção de liteira diversificada nos SAFs, juntamente com a adição de adubo verde das "cercas vivas" e das capinas e colheitas de palmito ou frutos da pupunha, cria uma variedade de micro-sitios favoráveis à presença e atividade da macrofauna.

\section{CONCLUSÕES}

Isopoda foi o grupo com a maior densidade e biomassa entre os macroinvertebrados da liteira, seguido pelos Diplopoda, nos SAFs, e pelos Isoptera (cupins), na capoeira. Estes últimos foram pouco encontrados na liteira dos SAFs. As maiores densidades e biomassas da macrofauna ocorreram nos tratamentos AS1 e AS2 (os mais diversificados), principalmente nos locais dominados pela liteira das palmeiras, do cupuaçu e dos adubos verdes nas entre-linhas, indicando que estas liteiras formam micro-sitios favoráveis ao abrigo e à ação decompositora da macrofauna.

Os sistemas agroflorestais diversificados contribuíram para a melhoria da biologia do solo, em todos os tratamentos estudados, quer pela produção de liteira de melhor qualidade, quer pela formação de micro-sítios com abundância de liteira de menor qualidade, como é o caso da pupunha e do cupuaçu, que formam locais apropriados para refúgio e ação da macrofauna. Em ambos os casos, a diversificação dos tipos de liteira, produzidos pelas espécies plantadas nos
SAFs, correspondeu também a uma maior diversidade, densidade e biomassa da macrofauna da liteira.

\section{AGRADECIMENTOS}

Os autores agradecem o auxílio financeiro do $\mathrm{CNPq} / \mathrm{INPA} / \mathrm{ORSTOM}$ (PPI 3130); à CPAA/EMBRAPA, pelo apoio no trabalho de campo e uso das áreas de estudo; à CEPLAC, pela cessão dos dados climáticos. À Michelle Costa e ao João Batista, pela ajuda nas coletas da macrofauna. À Kathrin Vohland, pela identificação das famílias dos diplópodos. À Eleusa Barros, pela ajuda na interpretação dos resultados da macrofauna e ao Dr. José W. Morais pelas valiosas sugestões dadas.

\section{Bibliografia citada}

Adis, J. 1997. Estratégias de sobrevivência de invertebrados terrestres em florestas inundáveis da Amazônia central. Acta Amazonica, 27(1): 43-54.

Adis, J.; Golovatch, S.I.; Hamann, S. 1996. Survival strategy of the terricolous millipede Cutervodesmus adisi Golovatch (Fuhrmannodesmidae, Polydesmida) in a blackwater inundation forest of Central Amazonia (Brazil) in response to the flood pulse. Acta Myriapodologica, Mém. Mus. Hist. Nat. 169: 523-532.

Anderson, J.M. 1988. Spatio-temporal effects of invertebrates on soil processes. Biol. Fertil. Soils, 6: 216-227.

Anderson, J.M.; Ingram, J. 1993. Tropical Soil Biology and Fertility. A Handbook of Methods, $2^{3}$ ed. C.A.B. International, Oxford, UK. 221 p.

Anderson, J.M.; Leonard, M.A.; Ineson, P.; Huish, S. 1985. Faunal biomass: A key component of a general model of nitrogen mineralisation. Soil Biol. Biochem.., 17: 735-737.

Anderson, J.M.; Swift, M.J. 1983. Decomposition in tropical rain forests. In: Sutton, S. L.; 
Chadwick, A. C.; Whitmore, T. C. (Eds). The Tropical Rain Forest: Ecology and Management. Oxford, UK: Blackwell. p, 287-309.

Bandeira, A.G.; Harada, A.Y. 1991. Cupins e Formigas na Amazônia. In: Val, A.L.; Figliuolo, R.; Feldberg, E. (Eds). Bases Cientificas para Estratégias de Preservação e Desenvolvimento da Amazônia: Fatos e Perspectivas. Vol. 1. Instituto Nacional de Pesquisas da Amazônia (INPA). Manaus, Amazonas. p. 387-395.

Bandeira, A.G.; Harada, A.Y. 1998. Densidade e distribuição vertical de macroinvertebrados em solos argilosos e arenosos na Amazônia central. Acta Amazonica 28(2): 191-204.

Cantarutti, R.B.; Urquiaga, S.; Boddey, R.M.; Braga, J.M.; Cassini, S.T. 1996. Cinética de mineralização do $\mathrm{N}$ contido na palha de Brachiaria humidicola e de Desmodium ovalifolium cv. Itabela. XIII Congresso Latino-americano de Ciência do Solo, 1996. CD ROM, artigo 03-086 (4 pág) Software Gráfico Ltda. Campinas, SP.

Coinneau, Y. 1974. Introduction à l'Étude des Microartropodes du Sol et de ses Anexes. Ed. Doin, Paris. 118p.

Correa, M.E.F.; Faria, S.M. 1997. Variaçâo sazonal da comunidade de macroartrópodos edáficos sob plantios de duas leguminosas arbóreas. XIII Congresso Latino-americano de Ciência do Solo, 1996. CD ROM, artigo 03-072 (4 pág.) Software Gráfico Ltda. Campinas, SP.

Dacaens, T.; Lavelle, P.; Jimenez Jaen, J.J.; Escobar, G.; Rippstein, G. 1994. Impact of land management on soil macrofauna in the Oriental Llanos of Colombia. Eur: J. Soil Biol., 30(4): 157-168.

Dindal, D.L. (Ed). 1990. Soil Biology Guide. John Wiley \& Sons. New York. 1349 p.

Edwards, C.A. 1974. Macroarthropods. In: Dickinson, D.H.; Pugh, G.J.F. (Eds). Biology of Plant Litter Decomposition. Academic Press, London. Vol. 1. p. 188-195.

EMBRAPA/CPAA. 1997. Relatório anual. Subprojeto 1: Dinâmica do Solo, da Vegetação e Efeitos Ambientais sob Sistemas Agroflorestais em Pastagens Degradadas. 19p.
Fernandes, E.C.M.; Matos, J.C.S. 1995. Agroforestry strategy for alleviating soil chemical constraints to food and fibre production in Amazon. In: Seidll, P.R.; Gottlieb, O.R.; Kaplan, M.A.C. (Eds). Chemistry of the Amazon. American Chemical Society, Series No. 588. ACS Books Dept., Washington, DC.

Fittkau, E.J.; Klinge, H. 1973. On biomass and trophic structure of the Central Amazonian rain forest, Biotropica, 5(1): 2-14.

Har. 'ר, A.Y.; Bandeira, A.G. 1994. Estratificação e densidade de invertebrados em solo argiloso sob floresta e plantios arbóreos na Amazônia central durante a estação seca. Bol. Mus. par. E. Goeldi, sér. Zool. 10: 235-251.

Hoffman, R.L.; Golovatch, S.I.; ADIS, J.; Morais, J.W. 1996. Practical keys to the orders and families of millipedes of the Neotropical region (Myriapoda: Diplopoda), Amazoniana, XIV $(1 / 2): 1-35$.

Hopkin, S.P.; Read, H.J. 1992. The Biology of Millipedes, Oxford Science Publications, $233 \mathrm{p}$.

ICRAF (International Centre for Research in Agroforestry). 1996. Annual Report. Nairobi, Kenya. p. 39-69.

Lavelle, P. 1988. Assessing the abundance and role of invertebrate communites in tropical soils: Aims and methods. In: Ghabbour, S. I.; Davis, R. C. (Eds). Proceedings of the Seminar on Resources of Soil Fauna in Egyppt and Africa. Cairo, 16-17 Abril 1986. Revue Zool.Afr-J. Afr: Zool., 102: 275-283.

Lavelle, P.; Chauvel, A.; Fragoso, C. 1995. Faunal activity in acid soils. Date, R. A. (Ed). Plant Soil Interactions at Low pH. p. 201-211.

Lavelle, P; Pashanasi, B. 1989. Soil macrofauna and land management in Peruvian Amazonia (Yurimaguas, Loreto). Pedobiologia, 33: 283-291.

Lawrence. R.F. 1953. The Biology of the Cryptic Fauna of Forests. Balkema, Cape Town.

Lips, J.M.; Duivenvoorden, J.F. 1996. Fine litter input to terrestrial humus forms in Colombian Amazonia. Oecologia, 108: 138-150.

Luizão, F.J. 1995. Ecological Studies in Contrasting Forest Types in Central Amazonia. $\mathrm{Ph}$. D. thesis. University of Stirling, 
Stirling, UK. 288p.

Luizào, F.J; Schubart, H.O.R. 1987. Litter production and decomposition in a terra-firme forest of Central Amazonia. Experientia, 43: 259-265

Luizão, R.C.C.; Bonde, T.A.; Rosswall, T. 1992. Seasonal variation of soil microbial biomass. The effects of clear-felling a tropical rainforest and establishment of pasture in the Central Amazon. Soil Biol.Biochem., 24: 805-813.

Luizão, R.C.C.; Luizão, F.J. 1991. Liteira e biomassa microbiana do solo no ciclo de matéria orgånica e nutrientes em terra firme na Amazónia Central. In: Val, A. L.; Figliuolo, R,; Feldberg, E. (Eds). Bases Cientificas para Estratégias de Preservação e Desenvolvimento da Amazônia: Fatos e Perspectivas. Vol. 1. Instituto Nacional de Pesquisas da Amazônia (INPA). Manaus, Amazonas. p. 65-75.

Mackerrow, A.J. 1992. Nutrient Stocks in Abandoned Pastures of the Central Amazon Basin Prior to and Following Cutting and Burning. MSc. thesis. North Carolina State University, Raleigh, USA, 116 p.

Marino, M.C.; Furtado, J.S.; De Vuono, Y.S. 1980. Glossário de Termos Usuais em Ecologia. 1" edição. Academia de Cièncias do Estado de São Paulo. 24: 97-138.

Matos, J.C.S.; Perin, R.; Souza, S.G.A.; Wandelli, E.V., Arco-Verde, M.; Fernandes, E.C.M. 1996. Alterações da fertilidade em Latossolo Amarelo sob diferentes sistemas agroflorestais em Manaus. In: Liyro de Resumos. XXII Reunião Brasileira de Fertilidade do Solo e Nutrição de Plantas. Manaus, AM. pp. 682-683.

Morais, J.W. 1995. Abundância, Distribuição Vertical e Fenologia da Fauna de Arthropoda de uma Região de Água Mista, Próxima de Manaus, AM. Tese de Doutorado. São Paulo, 226p.

Muchmore, W.B. 1990. Terrestrial Isopoda. In: Dindal, D. L. (Ed). Soil Biology Guide. p. 805-817.

Nutting, W.L. 1990. Insecta: Isoptera. In: Dindal, D. L. (Ed). Soil Biology Guide. p. 997-1032.

Oliveira, E.P.; Franklin, E. 1993. O efeito do fogo sobre a mesofauna do solo:
Recolonização em áreas queimadas. Pesq. Agropec. Bras., 28(3): 357-369.

Palm, C.A.; Sanchez, P.A. 1991. Nitrogen release from the leaves of some tropical legumes as affected by their lignin and polyphenolic contents. Soil Biol. Biochem., 23: 83-88.

Petersen, H.; Luxton, M. 1982. A survey of the main animal taxa of detritus food web. Oikos, 39(3): 293-294.

Raw, F. 1967. Arthropoda (except Acari and Collembola). In: Burges, A.; Raw, F. (Eds). Soil Biology. Academic Press, London. p. 323-362.

Ribeiro, M.N.G.; Adis, J. 1984. Local rainfall variability, a potential bias for bioecological studies in the Central Amazon. Acta Amazonica, 14(1/2): 159-174.

Ribeiro, M.O.A. 1994. Abundância, distribuiçào vertical e biomassa de artrópodos do solo em uma capoeira na Amazônia Central. Dissertação de Mestrado. INPA/UA, Manaus, 120p.

Rodrigues, J.M.G. 1986. Abundancia e distribuiçào vertical de Arthropoda do solo em capoeira de terra firme. Dissertação de Mestrado. INPA UA, Manaus, $80 \mathrm{p}$.

Sanchez, P.A. 1976. Properties and Managament of Soils in the Tropics. John Wiley; Sons. New York. $618 \mathrm{p}$.

Stork, N.E.; Brindell, M.J.D. 1993. Arthropod abundance in lowland rainforest of Seram. In: Edwards, 1.D.; Macdonald, A.A.; Proctor, J. (Eds). Natural History of Seram. Intercept Ltd. UK. p. 115-130.

Stork, N.E.; Eggleton, P. 1992. Invertebrates as determinants and indicators of soil quality. Amer. Jour: Altern. Agricul., 7: 38-47.

Tapiia-Coral, S.C. 1998. Macrofauna da liteira em sistemas agroflorestais iimplantados em áreas de pastagens abandonadas na Amazônia central. Dissertação de Mestrado. INPA/UFAM, Manaus, 98p.

Tapia-Coral, S.C.; Luizão, R.C.C.; Perin, R.; Luizão, F.J. 1997. Relação entre tratamentos agrossilviculturais implantados em solos degradados e as propriedades bioquímicas do solo. In: Livro de Resumos, MS-060. IX Congresso Brasileiro de Microbiologia. 11-15 
de novembro Rio de Janeiro. p. 223.

Teixeira, L.B.; Bastos, J.B. 1989. Nutrientes nos Solos de Floresta Primária e Pastagem de Brachiaria humidicola na Amazônia Central. Boletim de Pesquisa. EMBRAPA/ CPATU, №98, 31p.

Teixeira, L.B.; Schubart, H.O.R. 1988. Mesofauna do Solo em Áreas de Floresta e Pastagens na Amazônia Central. Boletim de PesquisaEMBRAPA/CPATU, № 95, 16p.

Vohland, K.; Schroth, G. 1998. Litter macrofauna in tropical land use systems of differing plant diversity and input levels: single-tree patterns and management implications. In: Abstracts of presentations and posters. Third Shift Workshop, March 15-19. Manaus, Amazonas. C42.

Walker, I.; Franken, W. 1983. Ecossistemas frágeis: A floresta da terra firme da Amazônia central. Ciencia Interamericana, 23: 9-21.

Wandelli, E.; Perin, R.; Souza, S.G.; Matos, J.C.S.; Souza, J.N.; Fernandes, E.C.M. 1997. Sistemas agroflorestais: Uma alternativa para recuperação das áreas de pastagens degradadas na Amazônia Ocidental. Anais do III Simpósio Nacional de Recuperação de Áreas Degradadas - SINRAD. 18-24 maio. Ouro Preto, MG. p. 487-493.

Zar, J.H. 1974. Bioestatistical Analysis. PrenticeHall, Inc., Englewood Cliffs, N. J. 718p. 
Apêndice 1. Lista de espécies plantadas nos tratamentos de sistemas agroflorestais (SAFs). Os símbolos dos tratamentos são os mesmos explicados na Tabela 1.

\begin{tabular}{llll}
\hline Nome vulgar & Nome científico & Familia & Tratamento \\
\hline Açai & Euterpe oleracea Martius & Arecaceae & AS1 \\
Capoeirão & Columbrina glandulosa Perkins & Rhamnaceae & AS1 \\
Cupuaçu & Theobroma grandiflorum (S) K. Schung & Sterculiaceae & AS1 e AS2 \\
Pupunha & Bactris gasipaes Kunth & Arecaceae & AS1 \\
Acerola & Malphigia emarginata L. & Malpighiaceae & AS2 \\
Araçá boi & Eugenia stipitata McVaugh & Mirtaceae & AS2 \\
Castanha-do-Brasil & Bertholletia excelsa H.B.K & Lecythidaceae & AS2 \\
Ingá & Inga edulis Martius & Mimosaceae & AS2 e ASPs \\
Jenipapo & Genipa americana L. & Rubiaceae & AS2 \\
Mamăo & Carica papaya L. & Caryocaraceae & AS2 \\
Maracujá & Passiflora edulis L. & Passifloraceae & AS2 \\
Mogno & Swietenia macrophylla King & Meliaceae & AS2 e ASPs \\
Paricá & Schizolobium amazonicum Ducke & Mimosaceae & AS2 \\
Teca & Tectona grandis Nursery & Verbenaceae & AS2 \\
Braquiarăo & Brachiaria brizantha (Höchst) Stapf & Gramineae & ASP1 \\
Desmódio & Desmodium ovalifolium Wall & Fabaceae & ASP1 e ASP2 \\
Quicuio da Amazỏnia & Brachiaria humidicola (Rendle) Schweinckt & Gramineae & ASP2 \\
Gliricidia & Gilicidia sepium (Jacq.) Walp & Ceasalpinaceae & Cerca viva \\
\hline
\end{tabular}


to Apêndice 2. Densidade média (ind/ $\mathrm{m}^{2}$ ) dos grupos da macrofauna encontrada nos sistemas agroflorestais (SAFs) e no controle (CAP), na estação chuvosa, com sua respectiva percentagem em relação ao total e sua classificação (Class.) ordinal nos SAFs e no controle. Os valores são médias de três blocos $(n=3)$. Os simbolos dos tratamentos são os mesmos explicados na Tabela 1 .

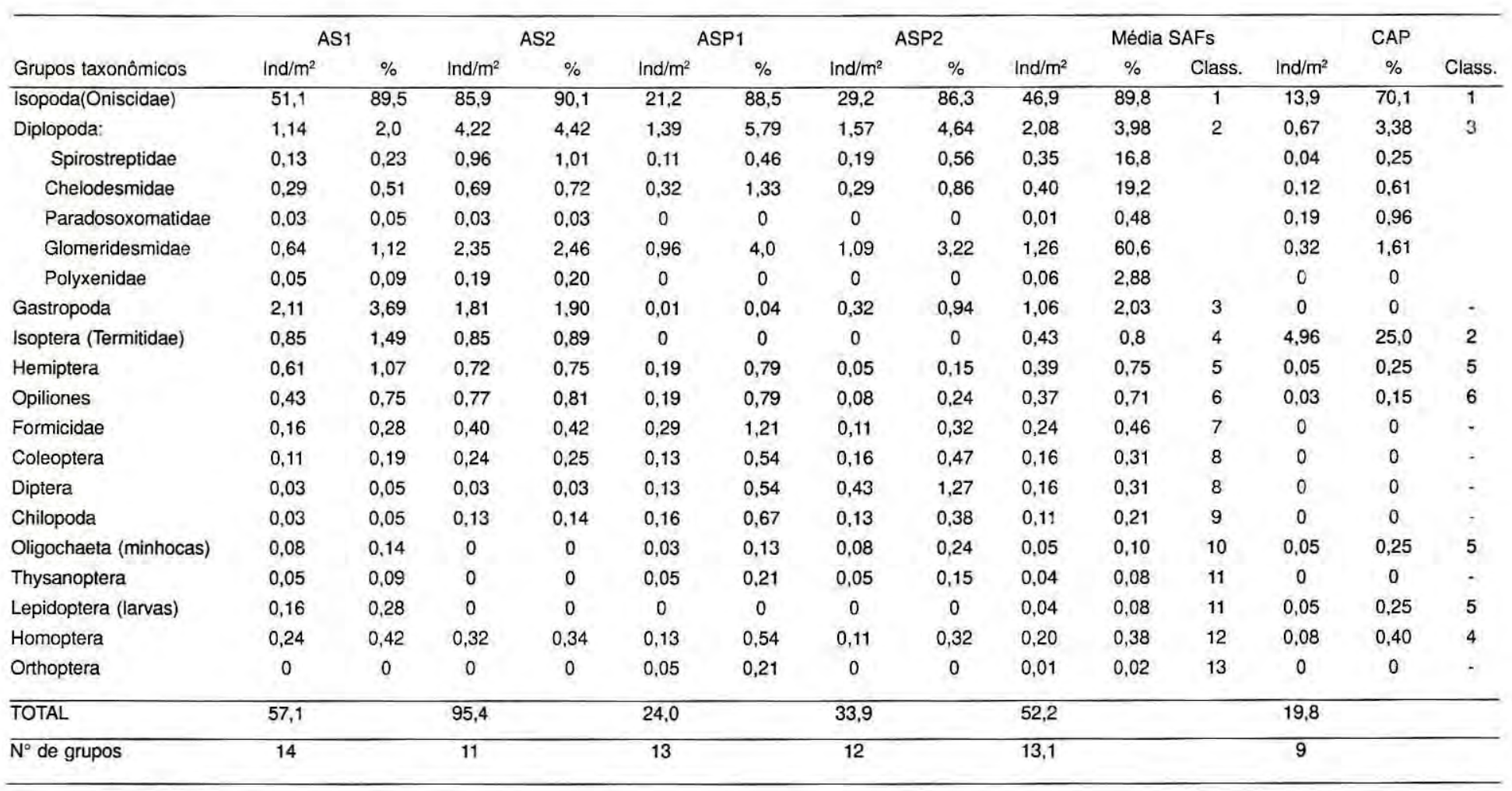


Apêndice 3. Densidade média (ind $/ \mathrm{m}^{2}$ ) dos grupos da macrofauna, encontrada nos sistemas agroflorestais (SAFs) e no controle (CAP), na estação seca, com sua respectiva percentagem em relação ao total e sua classificação (Class.) ordinal nos SAFs e no controle. Os valores são médias de três blocos $(n=3)$. Os simbolos dos tratamentos são os mesmos explicados na Tabela 1.

\begin{tabular}{|c|c|c|c|c|c|c|c|c|c|c|c|c|c|c|}
\hline \multirow[b]{2}{*}{ Grupos taxonômicos } & \multicolumn{2}{|c|}{ AS1 } & \multicolumn{2}{|c|}{ AS2 } & \multicolumn{2}{|c|}{ ASP1 } & \multicolumn{2}{|c|}{ ASP2 } & \multicolumn{3}{|c|}{ Média SAFs } & \multicolumn{3}{|c|}{ CAP } \\
\hline & $\ln d / m^{2}$ & $\%$ & $\operatorname{lnd} / \mathrm{m}^{2}$ & $\%$ & $\operatorname{lnd} / \mathrm{m}^{2}$ & $\%$ & $\operatorname{lnd} / \mathrm{m}^{2}$ & $\%$ & $\operatorname{lnd} / \mathrm{m}^{2}$ & $\%$ & Class. & $\operatorname{lnd} / \mathrm{m}^{2}$ & $\%$ & Class. \\
\hline Isopoda(Oniscidae) & 40,0 & 88,8 & 50,5 & 88,8 & 9,9 & 94,0 & 11,2 & 90,1 & 27,9 & 89,6 & 1 & 16,0 & 95,6 & 1 \\
\hline Isoptera(Termitidae) & 3,07 & 6,83 & 4,21 & 7,40 & 0 & 0 & 0,13 & 1,07 & 1,85 & 5,94 & 2 & 0,61 & 3,65 & 2 \\
\hline Gastropoda & 1,41 & 3,13 & 1,36 & 2,39 & 0,03 & 0,28 & 0,11 & 0,91 & 0,73 & 2,35 & 3 & 0 & 0 & - \\
\hline Coleoptera & 0,11 & 0,24 & 0,13 & 0,23 & 0,32 & 3,03 & 0,56 & 4,62 & 0,28 & 0,90 & 4 & 0,05 & 0,30 & 4 \\
\hline Diplopoda: & 0,22 & 0,48 & 0,24 & 0,42 & 0,03 & 0,28 & 0 & 0 & 0,12 & 0,39 & 5 & 0,06 & 0,36 & 3 \\
\hline Spirostreptidae & 0,11 & 0,24 & 0,21 & 0,37 & 0 & 0 & 0 & 0 & 0,08 & 66,67 & & 0,04 & 0,25 & \\
\hline Chelodesmidae & 0 & 0 & 0,03 & 0,05 & 0 & 0 & 0 & 0 & 0,01 & 6,25 & & 0,12 & 0,61 & \\
\hline Glomeridesmidae & 0,11 & 0,24 & 0 & 0 & 0 & 0 & 0 & 0 & 0,03 & 22,9 & & 0,32 & 1,61 & \\
\hline Polyxenidae & 0 & 0 & 0 & 0 & 0,03 & 0,28 & 0 & 0 & 0,01 & 6,25 & & 0 & 0 & \\
\hline Opiliones & 0,11 & 0,24 & 0,24 & 0,42 & 0,03 & 0,28 & 0,08 & 0,66 & 0,11 & 0,35 & 6 & 0 & 0 & - \\
\hline Hemiptera & 0,05 & 0,11 & 0,08 & 0,14 & 0,16 & 1,52 & 0,08 & 0,66 & 0,09 & 0,29 & 7 & 0 & 0 & - \\
\hline Homoptera & 0,03 & 0,07 & 0,05 & 0,09 & 0 & 0 & 0 & 0 & 0,02 & 0,06 & 8 & 0 & 0 & - \\
\hline Oligochaeta(minhocas) & 0,03 & 0,07 & 0,03 & 0,05 & 0 & 0 & 0 & 0 & 0,02 & 0,06 & 8 & 0 & 0 & - \\
\hline Formicidae & 0 & 0 & 0,03 & 0,05 & 0,03 & 0,28 & 0 & 0 & 0,02 & 0,06 & 8 & 0 & 0 & - \\
\hline Homoptera & 0 & 0 & 0 & 0 & 0,03 & 0,28 & 0 & 0 & 0,01 & 0,03 & 9 & 0 & 0 & - \\
\hline Thysanoptera & 0 & 0 & 0 & 0 & 0 & 0 & 0 & 0 & 0 & 0 & - & 0,03 & 0,18 & 5 \\
\hline Orthoptera & 0 & 0 & 0 & 0 & 0,03 & 0,28 & 0 & 0 & 0,01 & 0,03 & 9 & 0 & 0 & - \\
\hline TOTAL & 45,0 & & 56,9 & & 10,6 & & 12,1 & & 31,1 & & & 16,7 & & \\
\hline $\mathrm{N}^{\circ}$ de grupos & 9 & & 10 & & 8 & & 6 & & 7,78 & & & 5 & & \\
\hline
\end{tabular}

